\title{
Characterization of the mechanisms underlying the crosstalk between galectins and notch in gastric cancer
}

\author{
Sofia N Santos ${ }^{1}$, Celso Reis ${ }^{2}$, Marcelo Dias-Baruffi ${ }^{3}$, Roger Chammas $^{1}$, Emerson S Bernardes ${ }^{*}$ \\ From São Paulo Advanced School of Comparative Oncology \\ Águas de São Pedro, Brazil. 30 September - 6 October 2012
}

\section{Background}

Gastric cancer is the fourth most common cancer and the second leading cause of cancer-related deaths worldwide. Galectins form a family of $\beta$-galactosides binding proteins that recognize a variety of glycan-containing proteins at the cell surface and are overexpressed in various tumors, including gastric cancer. Galectins overexpression as well as changes in their subcellular distribution has been associated with gastric cancer progression and poor prognosis. It is not well understood, however, how the interaction between galectins and glycosylated receptors modulates tumor development and growth. Since Notch receptors and ligands contain glycan structures known to bind galectins, we aim to demonstrate that galectins expression in the tumor microenvironment may interfere with Notch signaling activation during tumor development and progression.

\section{Materials and methods}

Immunoprecipitation procedures with gastric cancer cell line AGS (ATCC CRL-1739) and MKN45 (ACC 409) were used to test for association between galectin-1/-3 and Notch-1 receptor. Furthermore, we transfected AGS cell line with siRNA against galectin-1/-3 or scramble using standard protocols (IDT DNA technologies), stimulate them with immobilized human recombinant delta-4 or Jagged-1 and assessed Notch-1 receptor activation.

\section{Results}

Galectin-1 and -3 interact with Notch-1 receptor and differentially modulate Notch signaling pathway upon activation by Delta/Jagged ligands. Galectin-1 knockdown alters Notch-1 activation induced by Delta- 4 whereas galectin-3 knockdown alters jagged-1-mediated Notch-1 activation. Furthermore, we found that exogenously added galectin-3 can enhance Notch-1 activation by Jagged-1.

\section{Conclusion}

Our results suggest that galectin- 1 and -3 interact with Notch-1 receptor and differentially modulate Notch signaling activation induced by Jagged-1 and Delta- 4 .

\section{Financial support \\ FAPESP and CAPES.}

\begin{abstract}
Author details
${ }^{1}$ Center for Translational Research in Oncology, Cancer Institute of the State of São Paulo, Brazil. ${ }^{2}$ Carcinogenesis Group, Institute of Molecular Pathology and Immunology of the University of Porto, Portugal. ${ }^{3}$ Faculty of

Pharmaceutical Sciences of Ribeirão Preto, University of São Paulo, Brazil.
\end{abstract}

Published: 4 April 2013

doi:10.1186/1753-6561-7-S2-P65

Cite this article as: Santos et al: Characterization of the mechanisms underlying the crosstalk between galectins and notch in gastric cancer. BMC Proceedings 2013 7(Suppl 2):P65.

\footnotetext{
* Correspondence: ebernardes@lim24.fm.usp.br

${ }^{1}$ Center for Translational Research in Oncology, Cancer Institute of the State of São Paulo, Brazil

Full list of author information is available at the end of the article
}

(c) 2013 Santos et al; licensee BioMed Central Ltd. This is an Open Access article distributed under the terms of the Creative Commons 